\title{
Optothermal dynamics in whispering-gallery microresonators
}

\author{
Xuefeng Jiang ${ }^{1}$ and Lan Yang (1)
}

\begin{abstract}
Optical whispering-gallery-mode microresonators with ultrahigh quality factors and small mode volumes have played an important role in modern physics. They have been demonstrated as a diverse platform for a wide range of applications in photonics, such as nonlinear optics, optomechanics, quantum optics, and information processing. Thermal behaviors induced by power build-up in the resonators or environmental perturbations are ubiquitous in high-quality-factor whispering-gallery-mode resonators and have played an important role in their operation for various applications. In this review, we discuss the mechanisms of laser-field-induced thermal nonlinear effects, including thermal bistability and thermal oscillation. With the help of the thermal bistability effect, optothermal spectroscopy and optical nonreciprocity have been demonstrated. By tuning the temperature of the environment, the resonant mode frequency will shift, which can also be used for thermal sensing/tuning applications. The thermal locking technique and thermal imaging mechanisms are discussed briefly. Finally, we review some techniques employed to achieve thermal stability in a high-quality-factor resonator system.
\end{abstract}

\section{Introduction}

In the last two decades, whispering-gallery-mode (WGM) microresonators ${ }^{1}$ have enabled numerous advances in fundamental science and technology, including optomechanics, non-Hermitian physics, communications, frequency combs, high-performance sensors, and cavity quantum electrodynamics $(\mathrm{QED})^{2-22}$. The capability of WGM microresonators to trap light in a highly confined volume for a long period of time significantly enhances light-matter interactions and enables a high-power build-up ${ }^{23,24}$. Consequently, thermal effects and the associated dynamics are ubiquitous in WGM microresonators. For example, thermooptic nonlinear dynamics ${ }^{25-28}$ and thermal instability ${ }^{29,30}$ have been observed in various applications. Specifically, temperature fluctuations affect the material refractive index and/or size of the resonator, both of which modify the mode distributions and shift the resonance frequencies of WGMs. Stable and continuous operation is critical for many applications, such as bio/chemical sensing ${ }^{31-39}$,

\footnotetext{
Correspondence: Lan Yang (yang@seas.wustl.edu)

${ }^{1}$ Department of Electrical and System Engineering, Washington University in St. Louis, St. Louis, MO 63130, USA
}

optomechanics $^{18,40-42}$, microlasers ${ }^{43-47}$, non-Hermitian physics $^{19}$, and nonlinear photonics ${ }^{2,3,48-54}$. Taking WGM sensing as an example, the resonance shifts induced by a target of interest ${ }^{34}$ are typically mixed with the thermally induced mode shift. Therefore, various thermal-stability techniques have been developed to suppress thermal noise in WGM microresonator applications, such as sensing and metrology, where thermally induced signal fluctuations are undesired. In addition, by making use of the thermal effects, innovative photonic techniques have been developed. For example, the thermal tuning technique has been applied to adjust the resonator frequency in parity-time (PT) symmetric resonator systems ${ }^{19,55,56}$ and to accurately measure the optothermal properties of resonators ${ }^{57,58}$. Furthermore, thermal sensing applications have also been demonstrated in many WGM microresonators using the thermo-optic effect and/or the thermal expansion effect. In addition, the thermal scanning technique has been demonstrated to reduce the noise of a frequency combs and optical solitons $^{59}$. Moreover, thermal locking ${ }^{60,61}$ and thermal imaging techniques ${ }^{62-64}$ have also been developed by taking advantage of thermal instability and thermal absorption, 
respectively, which will find broad applications in sensing, microscopy, and spectroscopy.

Here, in this review, the physical mechanism of the thermal nonlinear effects induced by probe/pump laser fields will first be discussed, including both the thermal bistability in a microresonator with a uniform material composition and the thermal oscillation in a hybridmaterial microresonator. Furthermore, optothermal spectroscopy, a thermal relaxation parameter measurement, and optical thermal nonreciprocity will also be introduced as applications of the thermal bistability effect. Second, thermo-optic applications, including thermal tuning, thermal scanning, thermal sensing, thermal locking and photothermal imaging techniques, will be discussed. Third, we will review some techniques to realize thermal stability. Finally, we offer a brief summary of thermo-optic dynamics and techniques in WGM microresonators.

\section{Thermal nonlinear effects}

Ultrahigh power build-up benefitting from a highquality factor $(Q)$ and small mode volume $(V)$ significantly enhances the absorption-induced thermal nonlinearity in WGM microresonators. Thermal bistability behavior typically occurs when scanning across a high- $Q$ mode, which is translated into linewidth broadening/narrowing behavior $^{26}$. In addition, a thermal oscillation may arise in a microresonator made of hybrid materials with different temperature coefficients.

\section{Thermal bistability}

In a WGM microresonator, the resonance frequency/ wavelength response to changes in temperature is affected by both the thermo-optic and thermal expansion effects. The thermo-optic effect $(\mathrm{d} n / \mathrm{d} T)$ represents the temperature dependence of the material refractive index, while the thermal expansion effect transfers temperature fluctuations into changes in the cavity size. Both the cavity size and refractive index can affect the resonance frequency according to the WGM resonance condition $(2 \pi n$ $(T) R(T)=m \lambda(T))$. The temperature of a WGM resonator can be tuned by an external thermal source or heat generated from optical absorption. External thermal sources have been widely applied to resonance frequency tuning and thermal sensing, which will be discussed later. In this section, we focus on the temperature change induced by the optical field itself. The resonance wavelength shift $(\Delta \lambda)$ as a function of a temperature change $(\Delta T)$ can be expressed as

$$
\Delta \lambda(\Delta T)=\lambda_{0}\left(\frac{1}{n} \frac{\mathrm{d} n}{\mathrm{~d} T}+\frac{1}{D} \frac{\mathrm{d} D}{\mathrm{~d} T}\right) \Delta T
$$

where $\lambda_{0}, D$ and $n$ are the resonance wavelength, effective diameter and refractive index of the cold cavity, respectively. $\mathrm{d} n / \mathrm{d} T$ and $(1 / D)(\mathrm{d} D / \mathrm{d} T)$ represent the coefficients of the thermal refraction and thermal expansion of the cavity, respectively.

The temperature dynamics can be described by ${ }^{26}$,

$$
C_{P} \Delta \dot{T}(t)=I \frac{1}{\left(\frac{\lambda_{p}-\lambda_{0}(1+\alpha \Delta T)}{\delta \lambda / 2}\right)^{2}+1}-K \Delta T(t)
$$

where $C_{p}$ and $K$ are the effective heat capacity and effective thermal conductivity of the cavity material, respectively. $I$ represents the optical power that actually heats the cavity, and $\delta \lambda$ is the mode linewidth of the cold cavity. The first term of Eq. (2) represents the heat generated from the resonant mode, while the second term represents the heat transfer to the environment.

The transmission spectrum of a silica microresonator appears as a triangular shape and a sharp dip during the wavelength up- and downscanning processes, known as thermal broadening and narrowing, respectively. Figure 1 shows the transmission, temperature, and resonance shift of a silica toroidal microresonator during the wavelength up- and downscanning processes ${ }^{26}$. Taking the wavelength

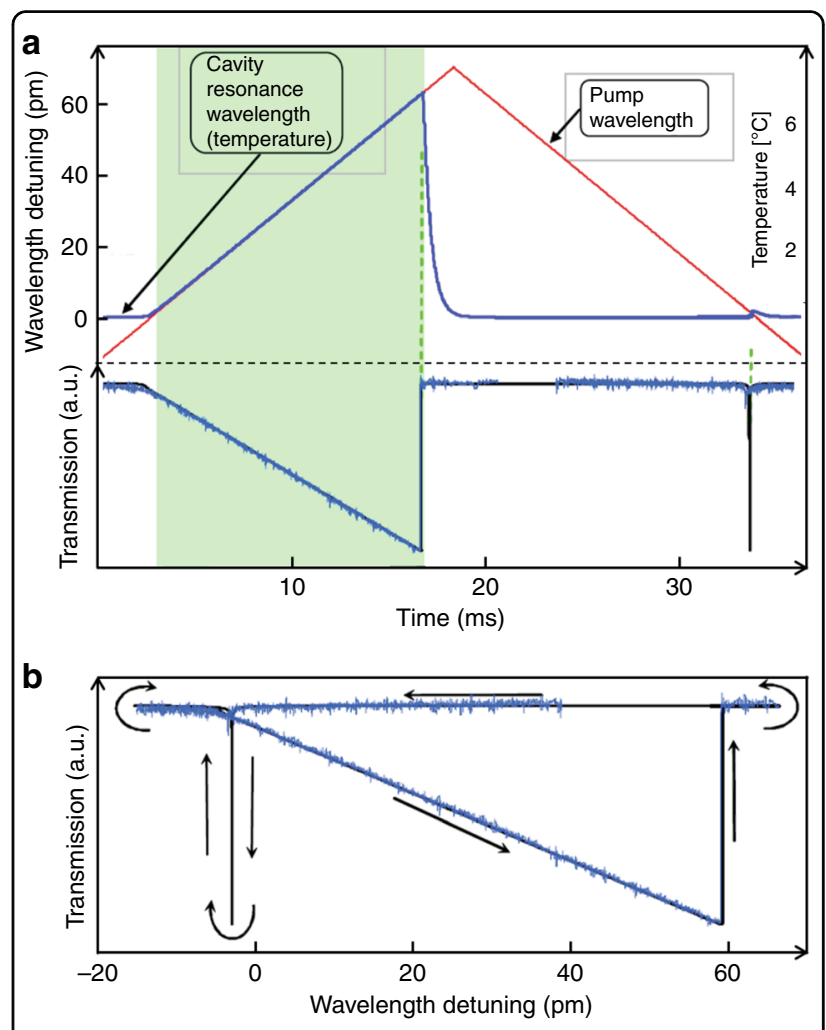

Fig. 1 Dynamic thermal bistability in a silica microtoroid resonator ${ }^{26}$. a Calculated transmission spectrum, cavity temperature, and resonance wavelength and the measured transmission spectrum during the wavelength up- and downscan processes. $\mathbf{b}$ Repeating transmission as a function of wavelength detuning in the upscanning (broadening) and downscanning (narrowing) processes. Reprinted with permission from ref. ${ }^{26}$ [OSA The Optical Society] 
upscanning process as an example, as the wavelength of the pump laser approaches the resonance wavelength (at $t$ $\sim 2 \mathrm{~ms}$ ), the cavity begins to heat up, which redshifts the resonance wavelength, making the upscan a "pursuit process" between the resonance wavelength and scanning pump wavelength; i.e., both the resonance wavelength and scanning pump wavelength shift in the same direction. Specifically, the pump follows the moving resonance wavelength, and the detuning between them decreases linearly; therefore, more pump is coupled into the resonator as the upscan proceeds. The pursuit process continues until the resonant point is caught up by the pump wavelength $(t \sim 16 \mathrm{~ms})$. At this point, the thermal absorption is maximal, and the compensation of the heat dissipation to the environment by thermal absorption can no longer be maintained. Beyond this point, the resonant state is rapidly lost, since the pump laser cannot shift the resonance wavelength further. On the other hand, in the case of the wavelength downscanning process, a narrowed resonance lineshape is observed due to an opposite process between the pump and resonance wavelengths; i.e., the thermally induced resonance shift and the scanning pump wavelength move in opposite directions, which results in passing through the resonance quickly.

As an application, thermal bistability can be used to scan the temperature of a WGM resonator during the wavelength upscanning process ${ }^{57}$. There is a linear region in Fig. 1a, where the temperature of the cavity increases linearly with time. This property can be used to thermally scan WGMs with a fixed-wavelength laser (Fig. 2b). Specifically, a high-power tunable laser in the $1440 \mathrm{~nm}$ wavelength band is used to pump and scan the temperature of the microresonator, while another fixedwavelength laser in the $1550 \mathrm{~nm}$ wavelength band is used to probe a high- $Q$ WGM when the resonance wavelength is thermally scanned. This thermal-bistability-assisted scanning scheme enables stable optothermal spectroscopy by removing thermal-noise-induced spectral perturbations, which could extend WGM applications to any wavelength band. Furthermore, optothermal spectroscopy has been used to control transmission spectra and optical gains in both erbium-doped ${ }^{65}$ and $\operatorname{Raman}^{66}$ WGM microlasers.

Recently, the thermal relaxation time and effective thermal conductance of a WGM could also be estimated by fitting two nearby optical modes modulated by the thermal effect ${ }^{58,67}$. Specifically, two nearby WGMs of a toroidal microresonator are scanned by a weak probe laser and a strong probe laser, where the weak probe records the wavelength detuning of the two WGMs; thus, the thermal relaxation time could be derived from the change in the detuning in the case of a strong probe. By tuning the strong probe power, the quantified thermal relaxation process could be fitted.

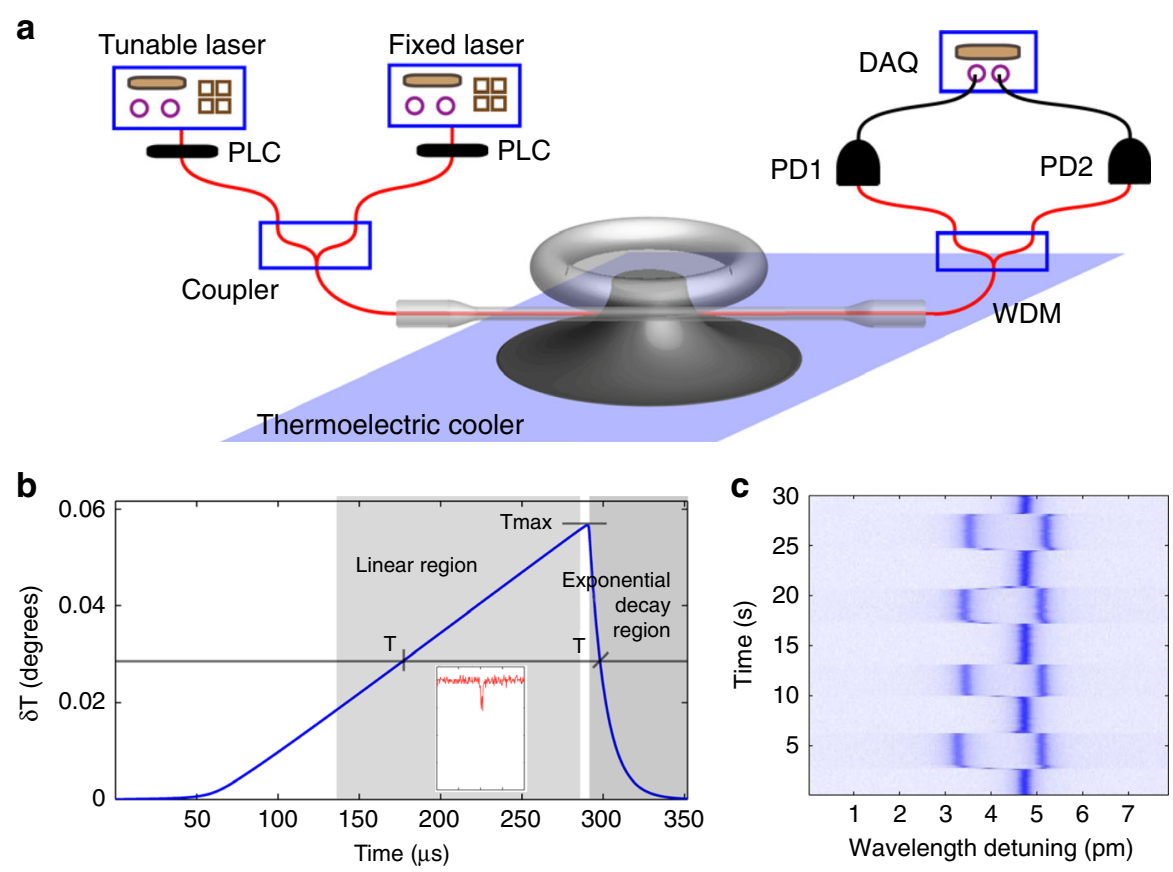

Fig. 2 Optothermal resonance scanning spectroscopy. a Schematics of the setup for optothermal spectroscopy. PLC polarization controller, PD photodetector, PM power meter, DAQ data acquisition, WDM wavelength division multiplexer. $\mathbf{b}$ Calculated temperature change of a high-Q mode during the wavelength upscanning process. Inset: transmission of a high-Q mode in the $1550 \mathrm{~nm}$ wavelength band scanned by a thermal pump WGM in the $1440 \mathrm{~nm}$ wavelength band ${ }^{50}$. c Transmission spectrum frames acquired by optothermal spectroscopy. Here, a nanofiber tip is repeatedly approaching and moving away from the microtoroid ${ }^{57}$. Reprinted with permission from ref. ${ }^{57}$ [American Institute of Physics] 
Optical nonreciprocal devices have attracted increasing attention in the past few years ${ }^{68}$. The standard method to realize optical nonreciprocity is through the magnetooptical effect, which requires an external magnetic field. Magnetic-free optical nonreciprocity in microresonators based on thermal nonlinear effects was proposed by Fan et al. ${ }^{69}$ Specifically, a silicon microring resonator coupled with two waveguides forms an add-drop filter system. The coupling strengths of these two waveguides are distinct due to the different ring-waveguide gaps. When exciting the resonator in opposite directions, the resonator undergoes different thermal broadenings in the transmission spectra. When choosing the resonant point as the operating wavelength, a nonreciprocal transmission ratio up to $40 \mathrm{~dB}$ could be achieved with this add-drop filter due to the thermal nonlinear effect.

\section{Thermal oscillation}

Hybrid microresonators can produce novel dynamic properties, such as thermal oscillation. Here, we discuss this phenomenon with a polydimethylsiloxane (PDMS)coated microtoroid as an example ${ }^{70}$. Figure 3 shows the experimental results and numerical simulations of dynamic changes in the transmission (Fig. 3b), temperature fluctuations in both the silica and PDMS layers (Fig. $3 c$ ), and the resonance wavelength in the wavelength upscanning process (Fig. 3d). There are four thermodynamic processes in the transmission spectrum, marked by regions with different colors.

\section{Region I}

From point $\mathrm{O}$ to $\mathrm{A}$ in Fig. 3b, the probe laser is coupled into the microresonator gradually when the probe wavelength $\left(\lambda_{\mathrm{p}}\right)$ approaches the resonance wavelength $\left(\lambda_{\mathrm{r}}\right)$. The temperature of the microresonator increases during this process due to thermal absorption, leading to a refractive index decrease/increase within the mode volumes in the PDMS/silica. As shown in Fig. 3c, the temperature increase in the PDMS layer is much larger than that in the silica layer, because the absorption coefficient of PDMS is much larger than that of silica in this wavelength band. Thus, the resonance is dominated by the thermo-optic effect of the PDMS layer, exhibiting a blueshift (green curve in Fig. 3d). On the other hand, considering that $\lambda_{\mathrm{p}}$ and $\lambda_{\mathrm{r}}$ shift in opposite directions, their detuning $(\Delta \lambda)$ decreases rapidly (purple curve in Fig. $3 \mathrm{~d}$ ), which gives rise to a rapid decrease in the transmission spectrum. Point A is the exact resonance frequency of the WGM $(\Delta \lambda=0)$, at which point the transmission decreases to the minimum value.

\section{Region II}

From point $\mathrm{A}$ to $\mathrm{B}$, the temperatures in both layers continue to increase. The resonance wavelength $\lambda_{\mathrm{r}}$

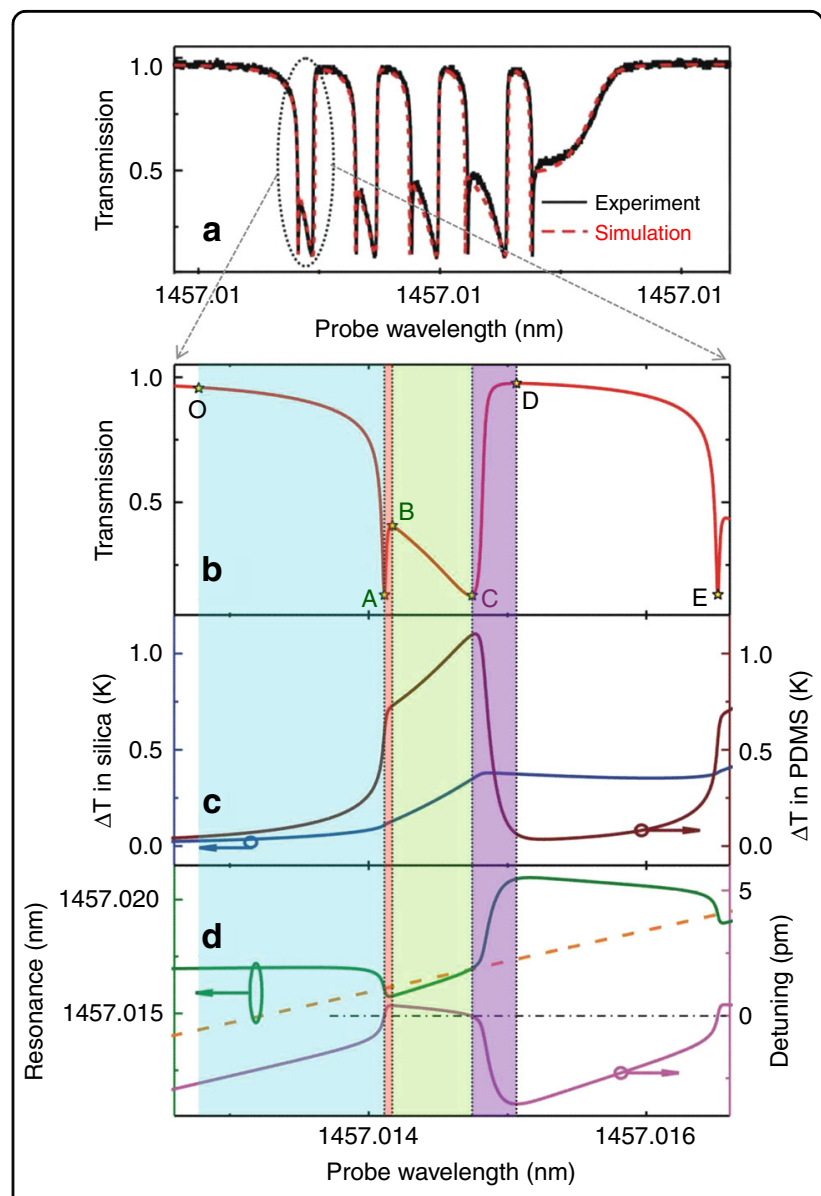

Fig. 3 Thermal oscillations in a PDMS-coated silica microtoroid during the wavelength upscanning process. a Measured and simulated optical transmission spectra. b Simulated transmission oscillation spectrum. c Simulated temperature dynamics in both silica and PDMS. d Calculated resonance wavelength $\left(\lambda_{r}\right)$, probe wavelength $\left(\lambda_{p}\right)$, and the associated variation in the detuning. Zero detuning is marked by a dot-dashed line (black) in $(\mathbf{d})^{70}$. Reprinted with permission from ref. ${ }^{70}$ [OSA The Optical Society]

exhibits a blueshift at the beginning due to the dominance of the PDMS. Considering that $\lambda_{\mathrm{p}}$ is larger than $\lambda_{\mathrm{r}}$ at point $\mathrm{A}$, the transmission increases rapidly with increasing $\Delta \lambda$ (Fig. 3b, d). The influence of the thermo-optic effect in the PDMS decreases as the optical absorption decreases, and $\lambda_{\mathrm{r}}$ undergoes a transition process from a blueshift to a redshift in this region. Although the resonance wavelength $\lambda_{\mathrm{r}}$ moves in the same direction as the wavelength upscanning process after the transition point, the speed of the redshift of $\lambda_{\mathrm{r}}$ cannot catch up with the wavelength upscanning process; thus, the transmission continues to increase. Note that the speed of the redshift of $\lambda_{\mathrm{r}}$ increases gradually. Finally, at point $\mathrm{B}$, the speed of the redshift of $\lambda_{\mathrm{r}}$ is the same as that of scanning $\lambda_{\mathrm{p}}$. As a result, at point $\mathrm{B}$, both the transmission and detuning $\Delta \lambda$ reach a local maximum point. 


\section{Region III}

From point $\mathrm{B}$ to $\mathrm{C}$, the thermo-optic effect in silica dominates due to the stable temperature increase in the silica section of the mode volume. Therefore, the speed of the redshift of $\lambda_{\mathrm{r}}$ is much faster than the speed for $\lambda_{\mathrm{p}}$, resulting in a decrease in both $\Delta \lambda$ and the transmission. At point $C, \lambda_{\mathrm{r}}$ equals $\lambda_{\mathrm{p}}$; thus, the transmission value reaches the local minimum a second time because the optical mode is on-resonance again.

\section{Region IV}

From point $C$ to $D$, the redshift of $\lambda_{\mathrm{r}}$ increases continually, resulting in an increase in both $\Delta \lambda$ and the transmission. Thus, the optical-absorption-induced heat decreases. When the optical absorption is smaller than the dissipation to the environment, the temperature in the mode volume starts to decrease. The temperature decreasing speed in PDMS is faster than the speed in silica, because the heat dissipation in PDMS is much larger. Thus, the dynamic behavior of $\lambda_{\mathrm{r}}$ is dominated by the thermo-optic effect of PDMS (green curve in Fig. 3d). The transmission increases quickly due to the fast separation of $\lambda_{\mathrm{r}}$ from $\lambda_{\mathrm{p}}$. Point $\mathrm{D}$ represents the nonresonant point, where the wavelength detuning is large enough, and thus the transmission returns to the maximum. After passing through point $\mathrm{D}$, a similar cycle repeats the whole process $(\mathrm{O}-\mathrm{A}-\mathrm{B}-\mathrm{C}-\mathrm{D})$, which gives rise to the thermal oscillation with a period from $\mathrm{O}$ to $\mathrm{D}$.

Thermal oscillations have been experimentally realized in not only microresonators with a coating, such as a PDMS-coated microtoroid $^{70}$, PDMS-coated microsphere $^{71}$, and PMMA-coated microtoroid ${ }^{72}$, but also uncoated microresonators with a uniform material composition such as a silica microsphere ${ }^{73,74}$, ZBLAN (ZrF4BaF2-LaF3-AlF3-NaF) microsphere ${ }^{75}$, millimeter-sized $\mathrm{BaF}_{2}$ disk $^{76}$, silicon nitride ${ }^{77}$, and lithium-niobate ${ }^{78,79}$ microdisk. The mechanisms of thermal dynamics in uncoated resonators are slightly different from those of coated resonators. They all result from the interplay of multiple effects with different influences on the resonance condition. For example, the thermal oscillation in a silica microsphere originates from the interplay between the Kerr nonlinear effect and the thermo-optic effect near $20 \mathrm{~K}^{74}$; the thermal oscillation in a ZBLAN microsphere is based on the interplay of three effects, including the Kerr effect, the thermo-optic effect, and thermal expansion ${ }^{75}$; the oscillation in $\mathrm{a} \mathrm{BaF}_{2}$ disk is due to the interactions of a positive thermo-optic effect, a negative thermoelastic effect, and the intrinsic Kerr nonlinearity ${ }^{76}$; and the oscillations in $\mathrm{Si}_{3} \mathrm{~N}_{4}$ and $\mathrm{LiNbO}_{3}$ microdisks result from the interplay of two nonlinear effects, i.e., a fast thermooptic nonlinearity and a slow process, such as a thermomechanical nonlinearity ${ }^{77}$, heat dissipation process $^{78}$ or photorefractive effect ${ }^{79}$. It should be noted that the frequency of the thermal oscillation is typically on the order of $\mathrm{Hz}$ to $\mathrm{kHz}$. However, Luo et al. report a $\mathrm{MHz}-$ level thermal oscillation in a PDMS-coated microsphere, which was considered to result from the competition between the thermo-optic effect and thermal-expansion effect of $\mathrm{PDMS}^{71}$.

\section{Thermo-optic applications \\ Thermo-optic tuning}

Tuning a WGM resonance is critical in many applications, such as PT symmetry ${ }^{19}$, tunable microlasers ${ }^{80-82}$, optical filters ${ }^{83}$, and cavity QED ${ }^{84,85}$. Various frequency tuning techniques have been explored, such as ther$\mathrm{mal}^{86,87}$, pressure/strain ${ }^{80-82,85,88-90}$, electro-optical ${ }^{91,92}$, magnetic-field $^{93}$, electrothermal ${ }^{94}$, internal aerostatic pressure $^{95,96}$, chemical etching ${ }^{97}$, and laser polishing ${ }^{98}$ techniques. Every technique has its own limitation. Specifically, electro-optical and electrothermal techniques are only applicable to special materials; pressure/strain and internal aerostatic pressure techniques are suitable for particular resonator structures, such as microbubbles; and etching and laser polishing techniques are disruptive, leading to permanent changes in the physical structures of the resonators. Among these techniques, thermal tuning is the simplest technique and essentially applies to all resonator structures/materials.

As an example, here we discuss a direct tuning method for a silicon microresonator that employs the thermooptic effect introduced by a visible laser diode ${ }^{99}$. As shown in Fig. 4a, the laser diode beam is focused on the top surface of the silicon microresonator. The resonance frequency shift as a function of the laser diode power is shown in Fig. 4b, which demonstrates a tuning rate of $0.0067 \mathrm{~cm}^{-1} / \mathrm{mW}$. It is worth noting that this direct thermo-optic tuning is a local and noninvasive method. In Fig. 4c, the temperature along the resonator depends on the angle $\theta$ for different pump positions, which affects the overlap of the laser beam with the resonant mode and leads to changes in the thermo-optic effect. Note that the local temperature when pumping at $\theta=\pi / 2$ is much larger than the temperature of the other two positions; this is attributed to the elliptic profile of the focused beam. In addition, the direct tuning method promises a much faster response than traditional thermal tuning methods. The normalized resonator response in Fig. 4d, defined as the normalized product of the pump power and the transmitted signal, is plotted as a function of the modulation frequency with a cut-off frequency of approximately $10 \mathrm{kHz}$. In the inset, the phase difference between the laser power and the transmitted light is presented, and the modulation depth of the signal as a function of the modulation frequency is also plotted. 
a

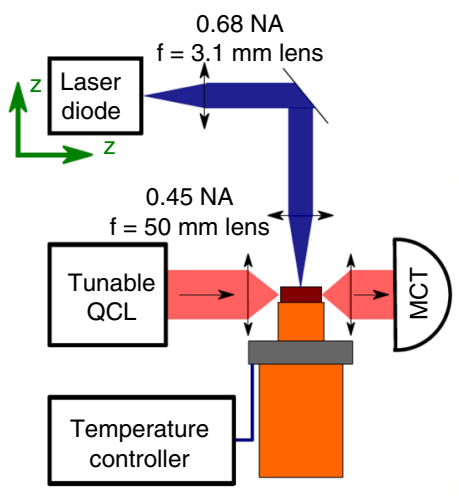

C

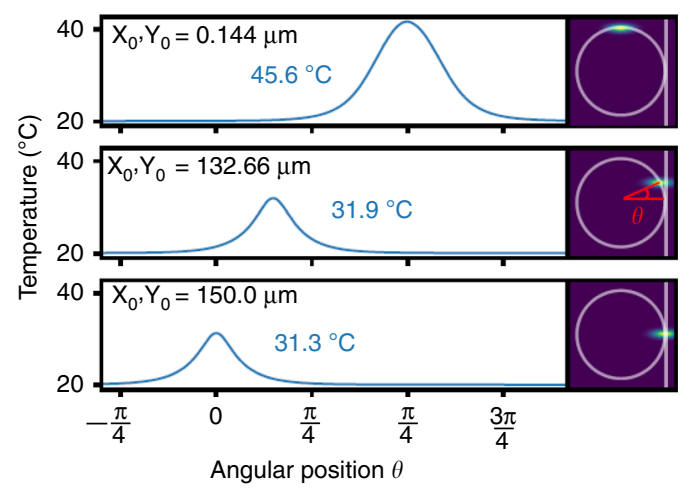

b

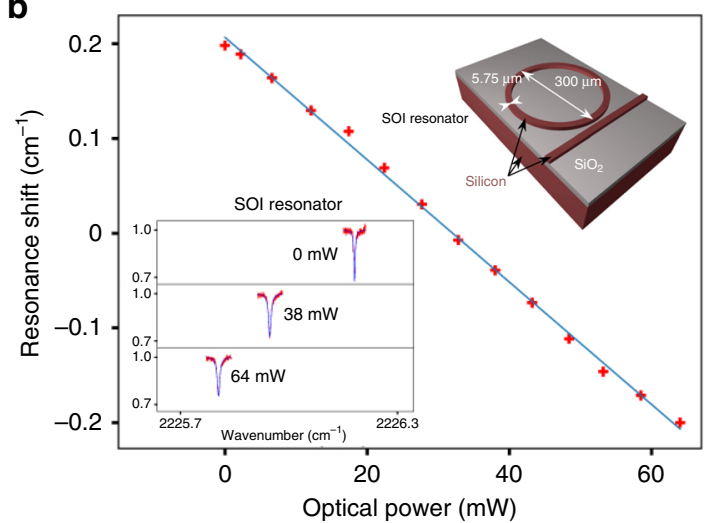

d

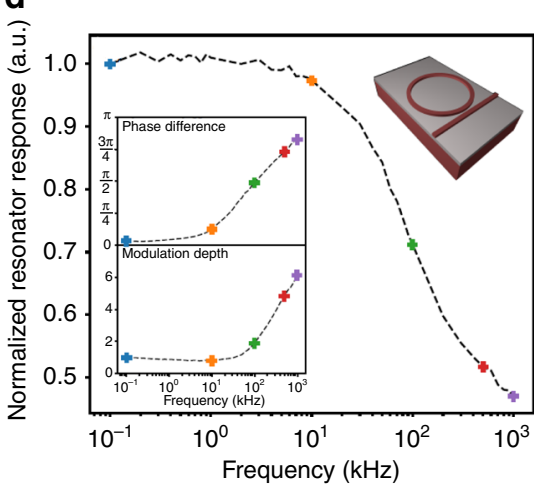

Fig. 4 Direct thermo-optic tuning of a silicon microresonator. a Schematic of the setup employed for direct thermo-optic tuning. $\mathbf{b}$ The resonance frequency is shifted while adjusting the optical power of the laser diode that serves as the pump to drive the thermo-optic effect. Bottomleft inset: the transmission signal at different optical powers, showing the resonance shift. Top-right inset: schematic of the device. $\mathbf{c}$ For a focused beam with dimensions of $w_{x} \sim 40 \mu \mathrm{m}$ and $w_{y} \sim 13 \mu \mathrm{m}$, the temperature profile along the angular position is plotted for three different beam positions. $\mathbf{d}$ The normalized response of the resonator as a function of the modulation frequency. Inset: the phase difference between the laser power and the detected mid-IR signal and the modulation depth of the mid-IR signal ${ }^{99}$. Reprinted with permission from ref. ${ }^{99}$ [OSA The Optical Society]

\section{Thermal scanning for comb and soliton applications}

Frequency comb generation and optical solitons, originating from cascaded four-wave mixing in a WGM microresonator, have attracted increasing interest in the past decade ${ }^{2,100-108}$. The generation of both a frequency comb and soliton typically relies on a tunable laser source, which has intrinsic drawbacks. Specifically, the linewidths of the best tunable lasers are usually on the order of $100 \mathrm{kHz}$, which is much broader than the linewidth of the best single-frequency laser. The broad linewidth and noisy amplitude of the tunable laser used for pumping limit the performance of the comb. In contrast, a single-frequency laser can provide much lower noise and a narrower linewidth than tunable lasers by using a reference frequency locking technique. For example, a narrow linewidth of $<40 \mathrm{mHz}$ has been demonstrated in a locked single-frequency laser ${ }^{109}$, which could be used as the pump source of a comb to reduce the noise on the generated comb lines.
Joshi et al. demonstrated a frequency comb and modelocked soliton with a single-frequency pump laser by thermally scanning the resonance frequency of the microresonator $^{59}$. As shown in Fig. 5a, an integrated platinum resistive microheater is fabricated on top of an oxide-clad $\mathrm{Si}_{3} \mathrm{~N}_{4}$ resonator. The resonance frequency could be controlled by tuning the current of the integrated microheater due to the refractive index change induced by the thermo-optic effect. A single-frequency laser with a linewidth of $\sim 1 \mathrm{kHz}$ is used as the pump laser, which is then amplified by an erbium-doped fiber amplifier (EDFA) and coupled into the on-chip bus waveguide through a fiber lens. The transmitted signals are monitored by a fast photoreceiver $(>12.5 \mathrm{GHz})$ and then analyzed by an optical spectrum analyzer, RF spectrum analyzer, and oscilloscope. By applying a triangular modulation to the heater current, the scanned transmission spectrum measured by the oscilloscope is shown in Fig. 5b, where the step-like structures marked by arrows indicate the transitions into 

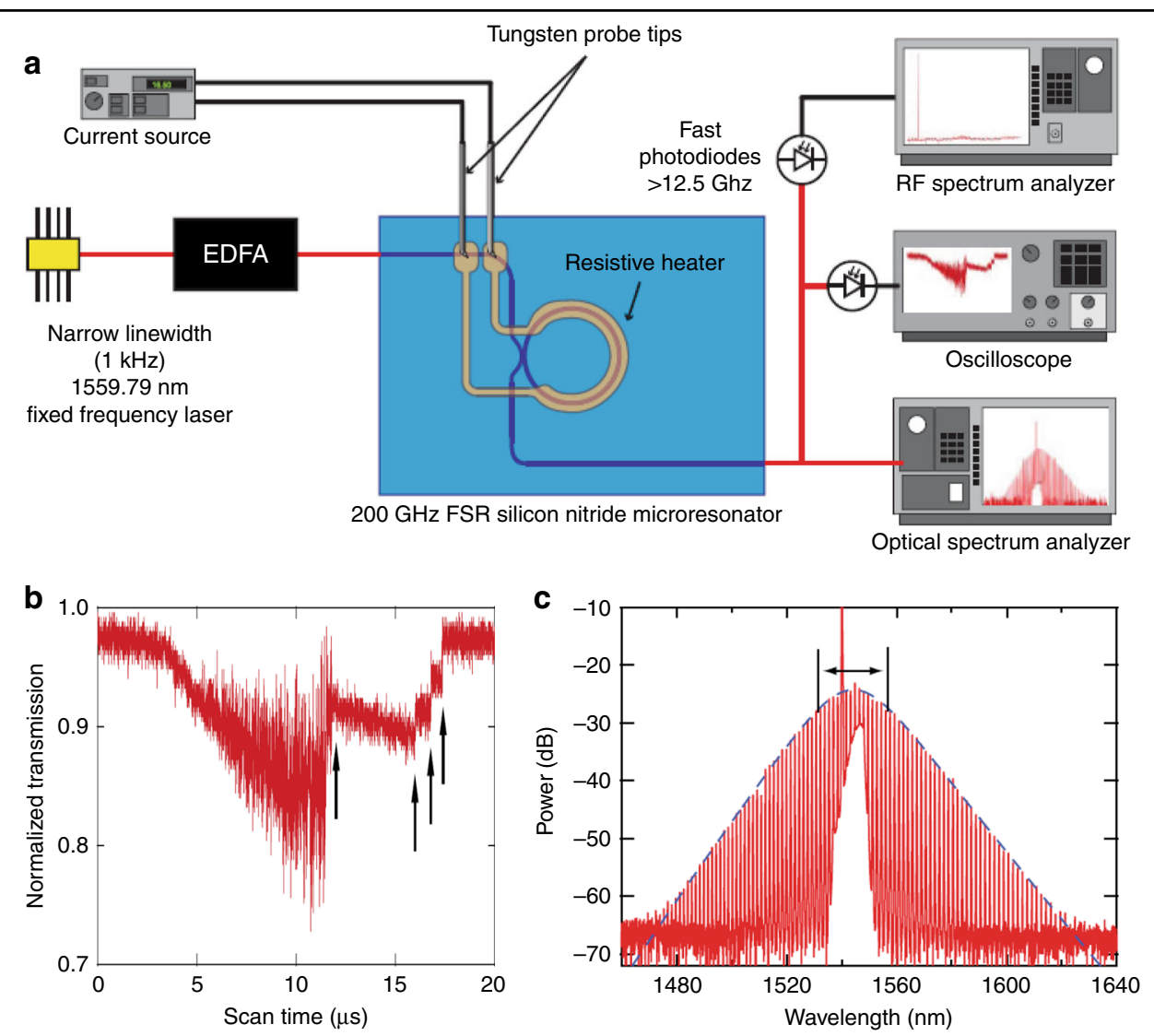

Fig. 5 Thermal scanning technique for soliton applications. a Schematics of the setup employed for the generation and characterization of optical solitons with the thermal scanning method. $\mathbf{b}$ Oscilloscope trace of the pump transmission as the current of the integrated heater is modulated with a triangular waveform. The steps indicated by the arrows are characteristic of transitions between different multisoliton states. c Measured optical spectrum for a single-soliton mode-locked state with the fitted sech ${ }^{2}$-pulse spectrum (blue dashed line) $)^{59}$. Reprinted with permission from ref. ${ }^{59}$ [OSA The Optical Society]

mode-locked soliton states ${ }^{110-112}$. Furthermore, the recorded optical comb spectrum agrees with the fitted sech $^{2}$ pulse spectrum (dashed blue curve in Fig. 5c), corresponding to a mode-locked single-soliton state.

\section{Thermal sensing}

A WGM microresonator can be utilized as a thermal sensor due to its ultrasensitive response to the temperature of the surrounding environment. In a WGM-based thermal sensor, the resonance wavelength/frequency is a linear function of both the refraction index and size of the WGM microresonator, both of which vary with the temperature of the environment due to the thermo-optic and thermal expansion effects, respectively. In principle, a cavity material with a larger thermo-optic coefficient and/ or thermal expansion coefficient typically leads to a larger frequency shift and thus allows for a more accurate temperature measurement.

Thermal sensing experiments have been demonstrated using silica- and silicon-based devices ${ }^{113-115}$. To improve the sensitivity of a thermal sensor, one can utilize a microresonator made of materials with larger thermooptic coefficients, such as PDMS ${ }^{116,117}$, UV-curable adhesives ${ }^{118}$, lithium niobate ${ }^{119}$, and dye-doped photoresists ${ }^{120}$, which give rise to a much better sensitivity. For example, a PDMS microsphere thermal sensor demonstrated a sensitivity of $0.245 \mathrm{~nm} / \mathrm{K}^{117}$. However, materials with large thermal expansion coefficients, such as silk, have also been used as a WGM thermal sensor. Specifically, a high thermal sensing sensitivity of $-1.17 \mathrm{~nm} / \mathrm{K}$ was realized in a silk fibroin microtoroid, which was attributed to the large thermal expansion effect ${ }^{121}$.

An alternative experimental design for temperature sensing involves the use of microdroplets, which can be made of a variety of materials, such as dye-doped cholesteric liquids, liquid crystals ${ }^{122}$, and oils ${ }^{123}$. The advantages of a microdroplet-based thermal sensor are the ease of integration with conventional microfluidics and diverse choices of materials with relatively high thermal refraction coefficients. Ward et al. also utilized thin-shelled microbubbles filled with air for temperature sensing ${ }^{124,125}$. Figure 6 summarizes the $Q$ factors and sensitivity of some 


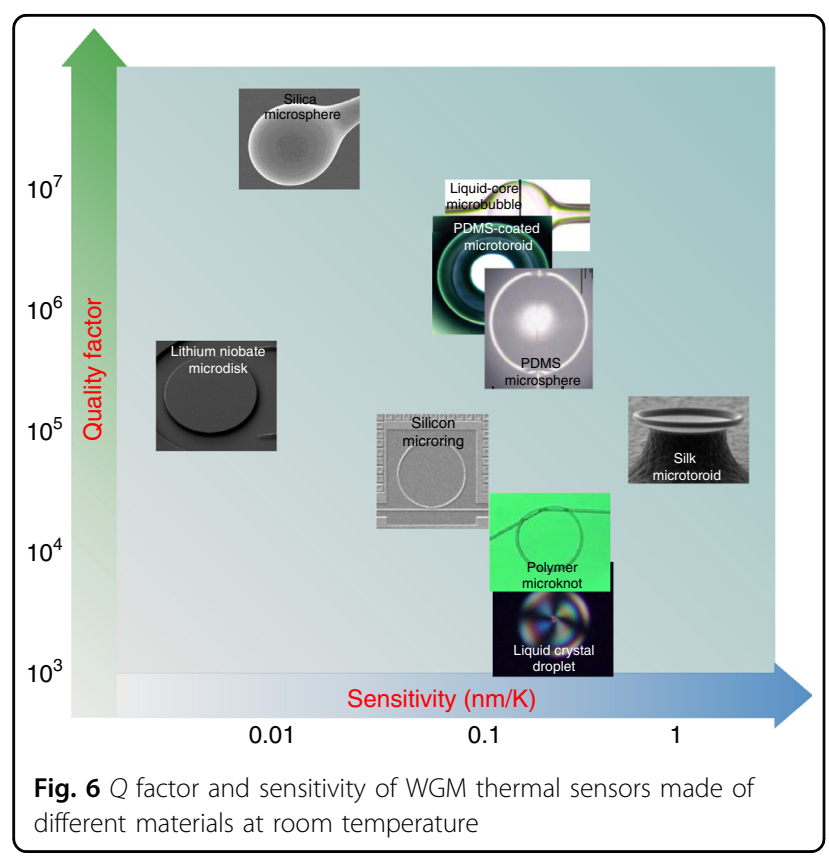

typical WGM thermal sensors operating at room temperature. Note that the silk microtoroid thermal sensor demonstrates the highest sensitivity $(1.17 \mathrm{~nm} / \mathrm{K})$, benefiting from the ultrahigh thermal expansion coefficient of the silk material ${ }^{121}$. It is worth mentioning that lithium niobate is an excellent candidate for self-referenced thermal sensing due to its strong thermo-optic birefringence. A preliminary study demonstrated self-referenced thermal sensing with an on-chip lithium niobate microdisk resonator with $Q \sim 10^{5}$ by using the different thermooptic responses of ordinary and extraordinary light in a birefringent material ${ }^{119}$. Experimentally, millimeter-sized lithium niobate WGM resonators ${ }^{126}$ with $Q$ factors up to $10^{8}$ and chip-based lithium niobate microdisks ${ }^{127}$ with $Q$ factors up to $10^{7}$ have been achieved, paving the way for the development of a high-performance thermal sensor.

\section{Thermal locking}

The Pound-Drever-Hall (PDH) locking technique has been widely used to lock the optical frequency of a probe/pump laser to a desired resonance frequency in various microresonator applications, such as optomechanics, nonlinear optics, and sensing ${ }^{128}$. In this method, a feedback voltage signal is applied to the laser head to lock the probe laser frequency to the resonance frequency or a frequency with a constant detuning from the resonance. As shown in Fig. 7a, a PDH error signal is generated by measuring the phase difference between the transmitted light and the probe laser ${ }^{60}$. A proportional-integral-derivative (PID) controller can read the laser's offset from the cavity resonance and feed this signal into a servo loop, which adjusts the frequency by minimizing the PDH error signal; the error signal is
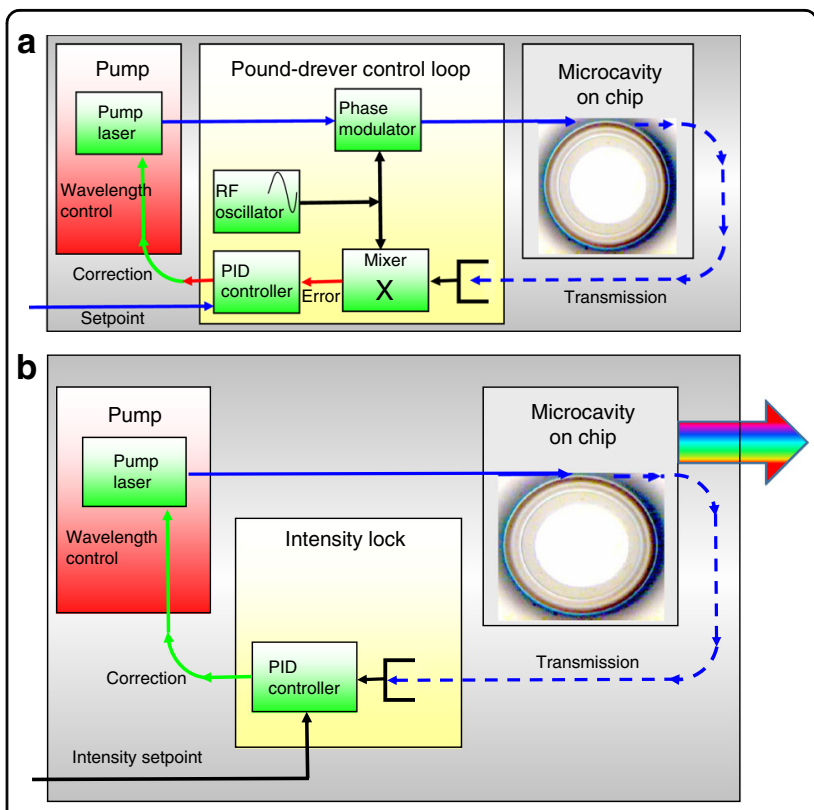

Fig. 7 Experimental setups of two locking systems. a

Pound-Drever-Hall (PDH) locking system. b Thermal locking system ${ }^{60}$. Reprinted with permission from ref. ${ }^{60}$ [OSA The Optical Society]

linear with the frequency detuning near the resonance, making the servo loop straightforward to manage.

Similarly, the thermally modified transmission spectrum during the wavelength upscanning process is nearly a linear function of the wavelength detuning, as shown in Figs. $1 \mathrm{a}$ and $2 \mathrm{~b}$, which can serve as an error signal for locking. Specifically, the linear region of the transmission function will be used as an input to the PID controller to stabilize the optical power coupled into the resonator, as shown in Fig. 7b. In this linear region, a small pump power increase (decrease) can heat up (cool down) the temperature of the resonator and thus increase (decrease) the frequency detuning by pushing the cavity resonance far away from (close to) the pump laser frequency. As a result, the optical absorption will decrease (increase). The whole process forms a negative feedback loop, which enables thermal locking ${ }^{60}$. It is worth noting that this thermal locking technique is only capable of locking to the resonance slope in the blue detuned region.

However, external environment perturbations may break the thermal locking status, which makes it difficult to achieve long-term locking. Therefore, to solve this problem, McRae et al. proposed another locking mechanism by combining the thermal locking technique with an optical locking technique, which could achieve long-term locking up to $12 \mathrm{~h}^{61}$. Specifically, the thermal locking technique is utilized to lock the resonance frequency of the microresonator to the probe laser frequency by optimizing the balance between optical absorption and thermal dissipation. An optical feedback locking system induced by scattering 

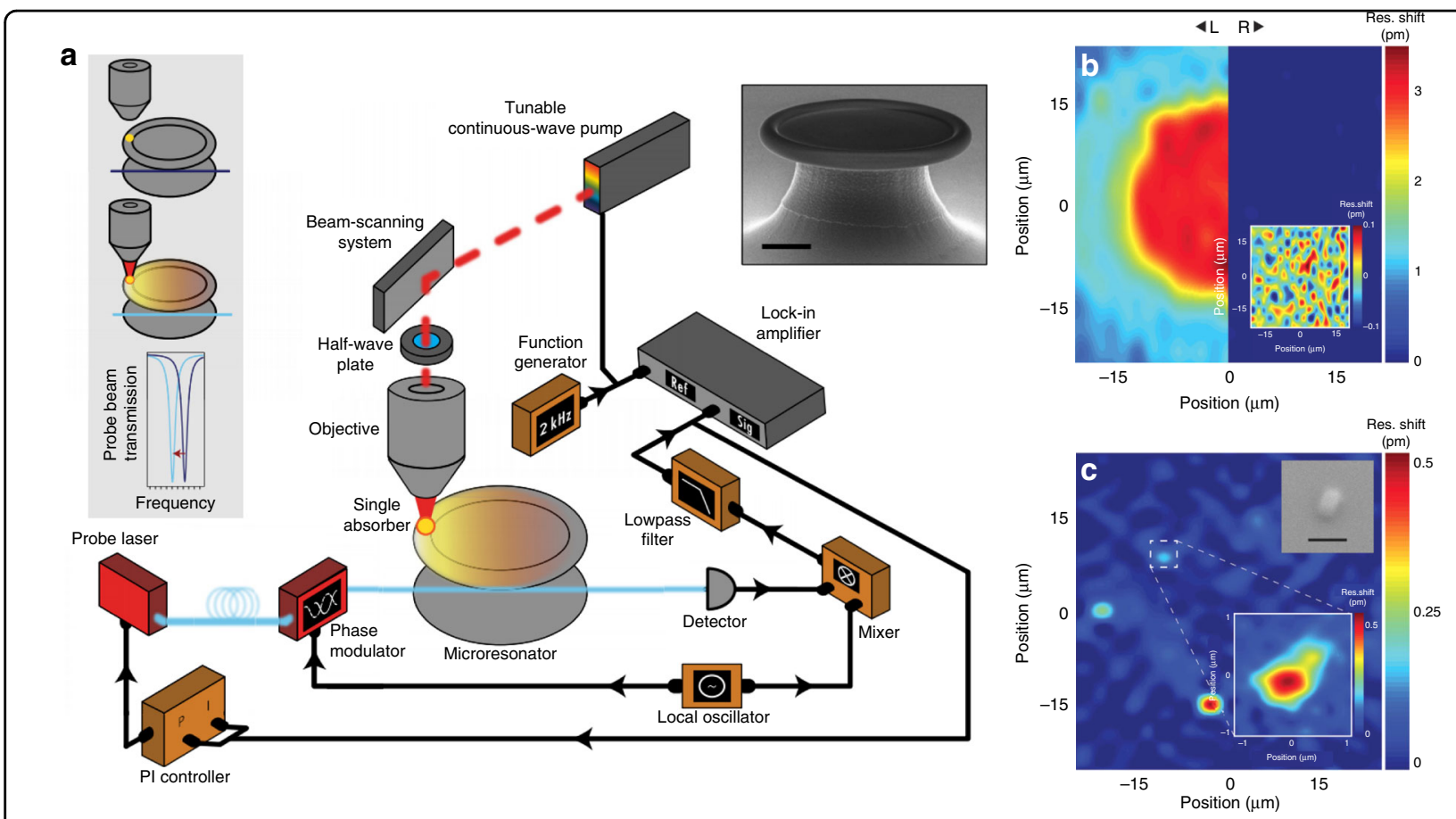

Fig. 8 Photothermal absorption spectroscopy and imaging. a Schematics of the setup employed for photothermal absorption spectroscopy and imaging. Inset (left): diagram of the thermal shift induced by the photothermal absorption of gold nanoparticles. Inset (right): scanning electron microscopy (SEM) image of a silica microtoroid. Scale bar: $10 \mu \mathrm{m}^{62}$. b Large-area maps of a silica-on-silicon toroid (left) and an all-glass toroid (right). c Photothermal imaging of a single gold nanorod on an all-glass microtoroid. Inset (top): SEM image of a nanorod with a scale bar of $100 \mathrm{~nm}$. Inset (bottom): high-resolution photothermal imaging of the nanorod ${ }^{64}$. a Reprinted with permission from ref. ${ }^{62}$ [Nature Publishing Group]; $\mathbf{b}$, $\mathbf{c}$ Reprinted with permission from ref. ${ }^{64}$ [John Wiley \& Sons, Inc.]

centers is utilized to achieve fast feedback control. The system locks the probe laser frequency to the resonance frequency by maximizing the constructive interference between the intracavity field and the feedback laser field.

\section{Photothermal absorption spectroscopy and imaging}

Previous studies are based on the thermal dynamics of the whole cavity or materials in the mode volume, while small temperature changes in the local environment of microresonators can also be detected by the thermal shift of the resonance, which is a similar effect used in photothermal absorption spectroscopy ${ }^{62}$. As shown in the left inset of Fig. 8a, high-Q WGMs in a microtoroid are excited through a tapered fiber. To perform photothermal absorption spectroscopy, a second free-space beam is focused on the surface of the microresonator, where a single gold nanoparticle is deposited. The excitation of the gold nanoparticle gives rise to a local temperature increase due to photothermal absorption, which redshifts the resonance. Photothermal absorption spectroscopy can be achieved by tuning the pump laser wavelength. A PDH locking system can be applied to the probe laser to improve the thermal shift sensitivity down to a single attometer. It should be noted here that an all-glass microtoroid (the right inset of Fig. 8a) is used to perform the photothermal absorption spectroscopy experiment to reduce the photothermal background noise of the silicon pillar of a normal silica-on-silicon toroid in the visible region.

On the other hand, photothermal absorption imaging can also be achieved by scanning the pump beam across the surface of the microresonator, as shown in Fig. 8b, $c^{62-64}$. In the experiment, mode shift signals are recorded in real time when scanning the pump laser beam spatially on the top surface of the resonators, which creates a thermal shift image. Experimentally, large-area thermal maps of a normal silica toroid on a silicon pillar (left) and an all-glass toroid (right) are recorded to compare the background noise (Fig. 8b). The background noise of the photothermal image for the all-glass microtoroid is much lower than that of the normal toroid on a silicon pillar at $630 \mathrm{~nm}$, which represents a great advantage of the all-glass microtoroid resonator as a platform for photothermal imaging. Furthermore, individual gold nanorods with a size of $40 \mathrm{~nm}$ by $80 \mathrm{~nm}$ are deposited onto the all-glass microtoroid, which are then imaged by thermal shifts. Experimentally, large-area photothermal imaging is first utilized to find the position of the nanorod on the microtoroid (Fig. 8b). High-resolution thermal imaging is then performed to image individual nanorods, as shown in Fig. 8c, with an SEM image presented in the top inset. 

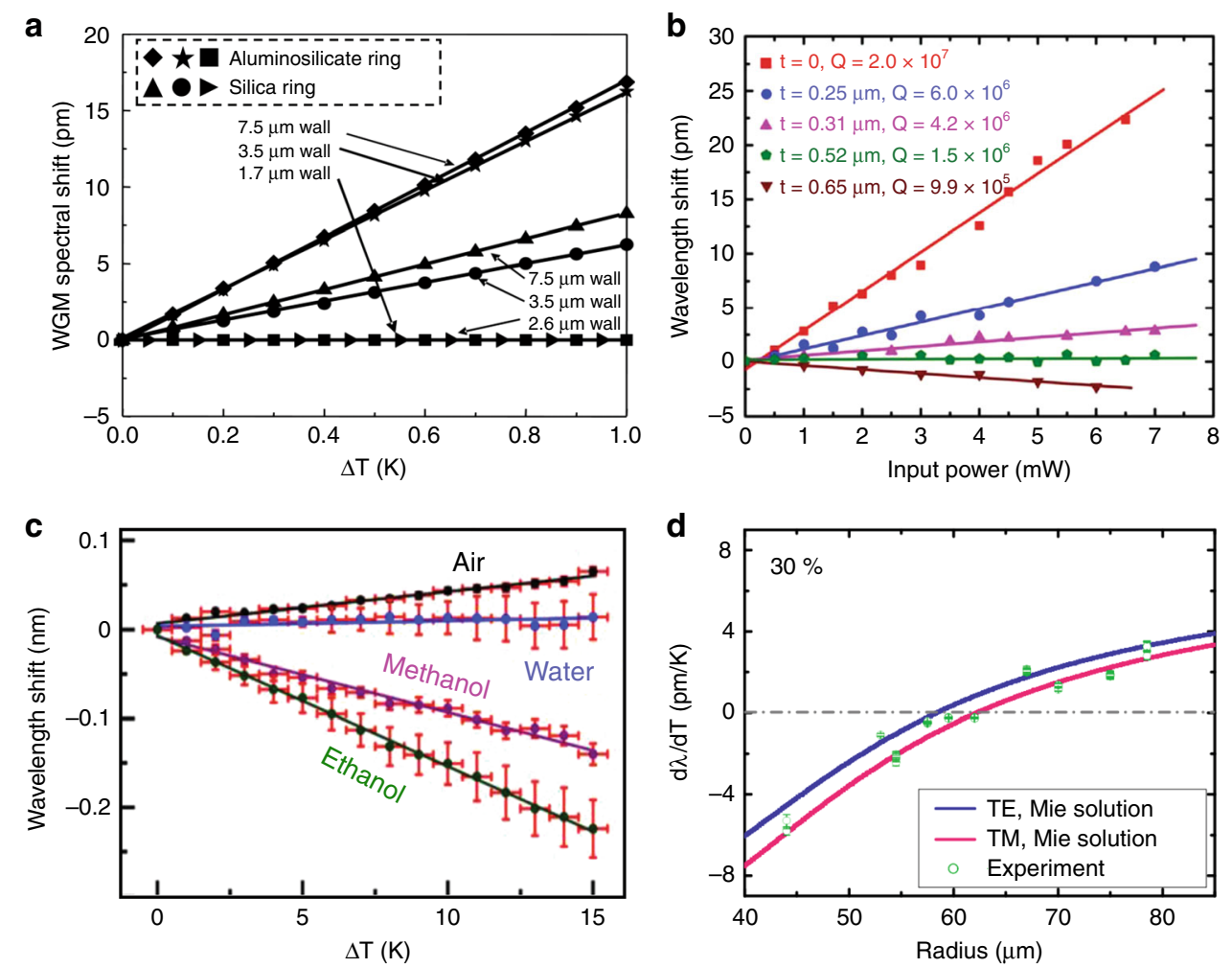

Fig. 9 Thermo-optic compensation in WGM microresonators. a Thermal sensitivity reduction by optimizing the geometry of liquid core aluminosilicate and silica optical rings ${ }^{129}$. b-d Thermo-optic compensation of WGM microresonators by coating with PDMS (b) $)^{131}$, a quantum dot $(\mathbf{c})^{133}$, and glycerol content in the surrounding aqueous medium $(\mathbf{d})^{134}$. a Reprinted with permission from ref. ${ }^{129}$ [OSA The Optical Society]. b Reprinted with permission from ref. ${ }^{131}$ [American Institute of Physics]. c Reprinted with permission from ref. ${ }^{133}$ [American Institute of Physics]. d Reprinted with permission from ref. ${ }^{134}$ [American Institute of Physics]

\section{Thermal stabilization}

The thermal stability of the WGM resonance is of great importance in almost all applications, such as lasing, optical sensing, optomechanics, nonlinear optics, and imaging. Various techniques have been developed to eliminate or reduce thermal noise to achieve thermal stabilization, which will be summarized in this section.

\section{Thermo-optic coefficient compensation}

Microresonators immune to thermal fluctuations have been fabricated by either optimizing the resonator structures/geometries or materials. For example, in a liquid core optical ring resonator, an optimization of the resonator's geometry with respect to the surrounding medium can facilitate a reduction in the WGM's thermal sensitivity. As shown in Fig. 9a, liquid core optical rings with a wall thickness of $1.7 \mu \mathrm{m}$ for an aluminosilicate microring and $2.6 \mu \mathrm{m}$ for a fused silica microring demonstrate minimum susceptibilities to temperature fluctuations ${ }^{129}$.

Hybrid microresonators fabricated by materials with opposite thermo-optic coefficients can also realize thermal stabilization. Theoretically, the thermal shift of WGMs in a silica microsphere with a positive thermo- optic coefficient could be compensated by coating a thin layer of a soft material with an opposite thermo-optic coefficient onto the surface of the microsphere ${ }^{130}$. He et al. demonstrated that a thin layer of PDMS coated on a silica microtoroid resonator could eliminate the thermal shift and broadening due to the thermo-optic compensation enabled by the opposite thermo-optic coefficients (Fig. 9b) ${ }^{131}$. Similar experiments were also performed with KD-310 glue ${ }^{132}$ and quantum $\operatorname{dot}^{133}$-coated microspheres (Fig. 9c). In addition, compensation of the thermal effect in a microsphere was also demonstrated by optimizing the glycerol content in the surrounding aqueous medium (Fig. 9d) ${ }^{134}$. The same stabilization scheme was utilized to characterize the thermal response of various polymer layers and protein molecules adsorbed to the resonator's surface.

\section{Thermal locking stabilization technique}

As mentioned previously, optothermal spectroscopy using thermal bistability has been adopted to eliminate the spectral perturbations caused by temperature fluctuations ${ }^{57}$. Here, we introduce another active thermal stabilization technique assisted by the thermal locking of 
a

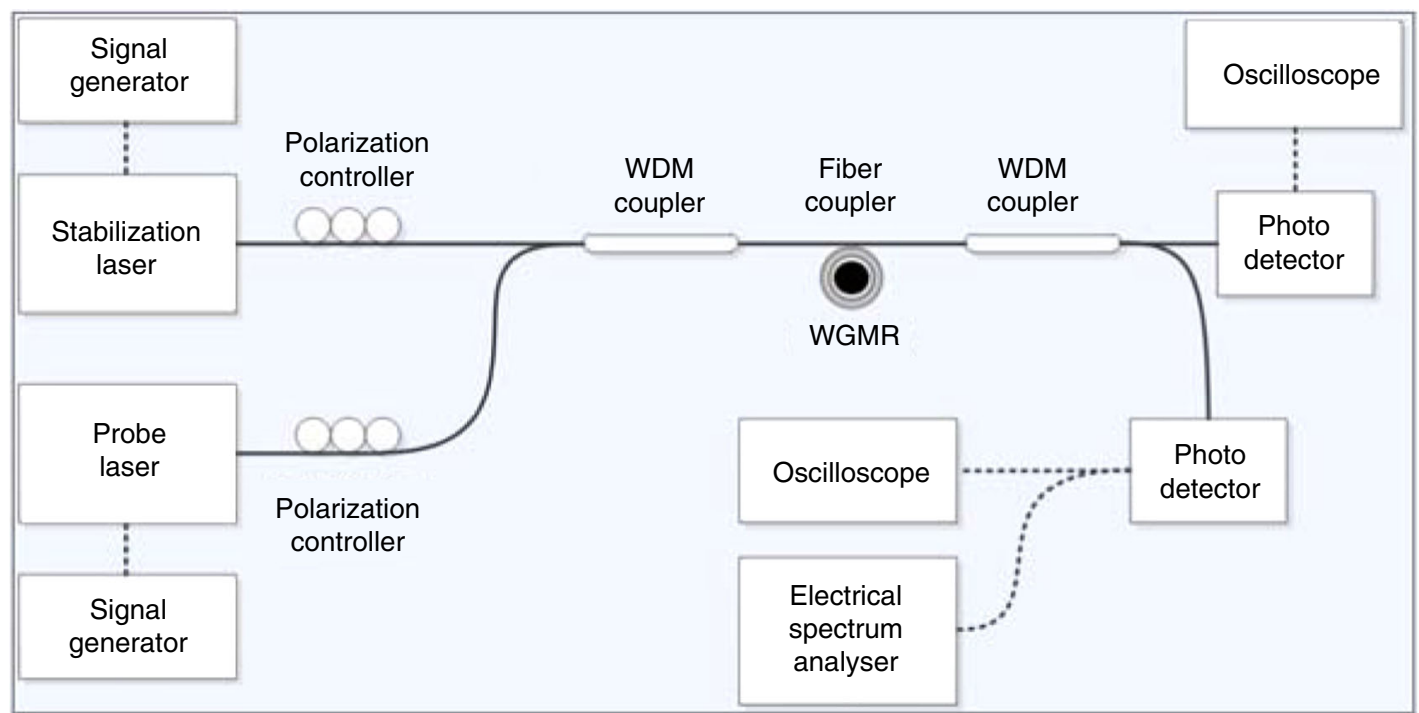

b

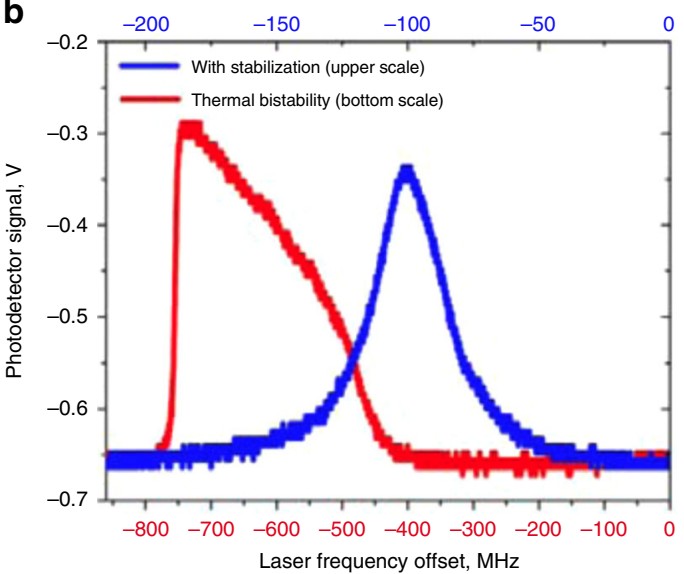

C

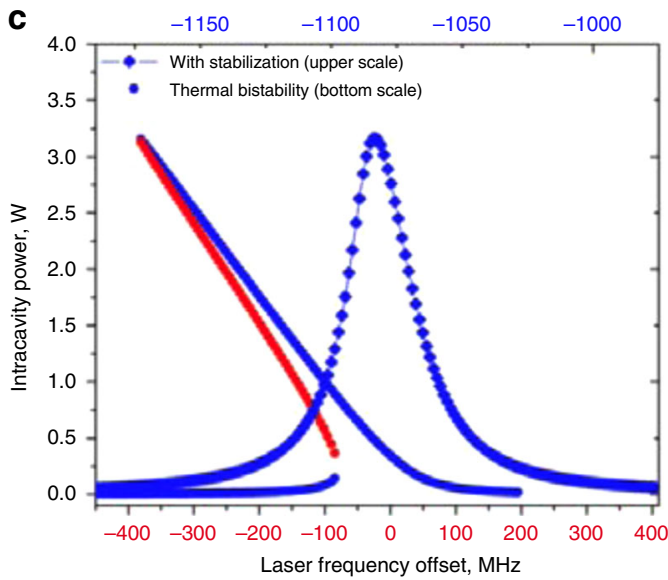

Fig. 10 Thermal locking thermal stabilization technique. a Schematic of the thermal locking stabilization setup. $\mathbf{b}$ Experimental spectrum of a high-Q WGM with (blue) and without (red) thermal locking stabilization. c Simulation results of a high-Q WGM with (blue) and without (red) thermal locking stabilization ${ }^{135}$. Reprinted with permission from ref. ${ }^{135}$ [OSA The Optical Society]

an additional laser ${ }^{135}$. Two WGMs with different resonance wavelengths and similar mode volumes were exploited in this approach. As shown in Fig. 10a, two tunable lasers are used: one laser operates in the $1550 \mathrm{~nm}$ band as a pump laser, and the other laser operates in the $1450 \mathrm{~nm}$ band, serving as a probe laser. Two WDM couplers are utilized to combine the pump and probe lasers and then separate the transmitted signals. A fiber taper waveguide is used to couple light into WGMs of a microtoroid. In the experiment, the WGM in the $1450 \mathrm{~nm}$ wavelength band is first scanned and recorded; the thermal nonlinearity is shown as the red curve in Fig. 10b. Then, the pump laser at $1550 \mathrm{~nm}$ is applied and thermally locked to the linear region of the second high- $Q$ mode in the $1550 \mathrm{~nm}$ wavelength band. Heating induced by the $1550 \mathrm{~nm}$ laser shifts the probe mode in the $1450 \mathrm{~nm}$ wavelength band. With the help of this thermal locking stabilization technique, the probe WGM in the $1450 \mathrm{~nm}$ wavelength band exhibits a perfect Lorentzian lineshape, as shown in Fig. 10b (blue curve). In addition, Fig. 10c shows the simulation results, which fit very well with the corresponding experimental data.

\section{Self-referenced dual-mode stabilization technique}

Another scheme employed to stabilize the resonator temperature is the self-referenced dual-mode temperature stabilization technique based on a simultaneous readout of TE and TM WGMs. Specifically, the resonance frequencies of TM and TE WGMs in a microresonator have different temperature coefficients; thus, the selfreferenced mode shift difference between the modes can serve as a perfect temperature sensing signal, which can also be utilized for active stabilization of the cavity temperature $^{136-139}$. 

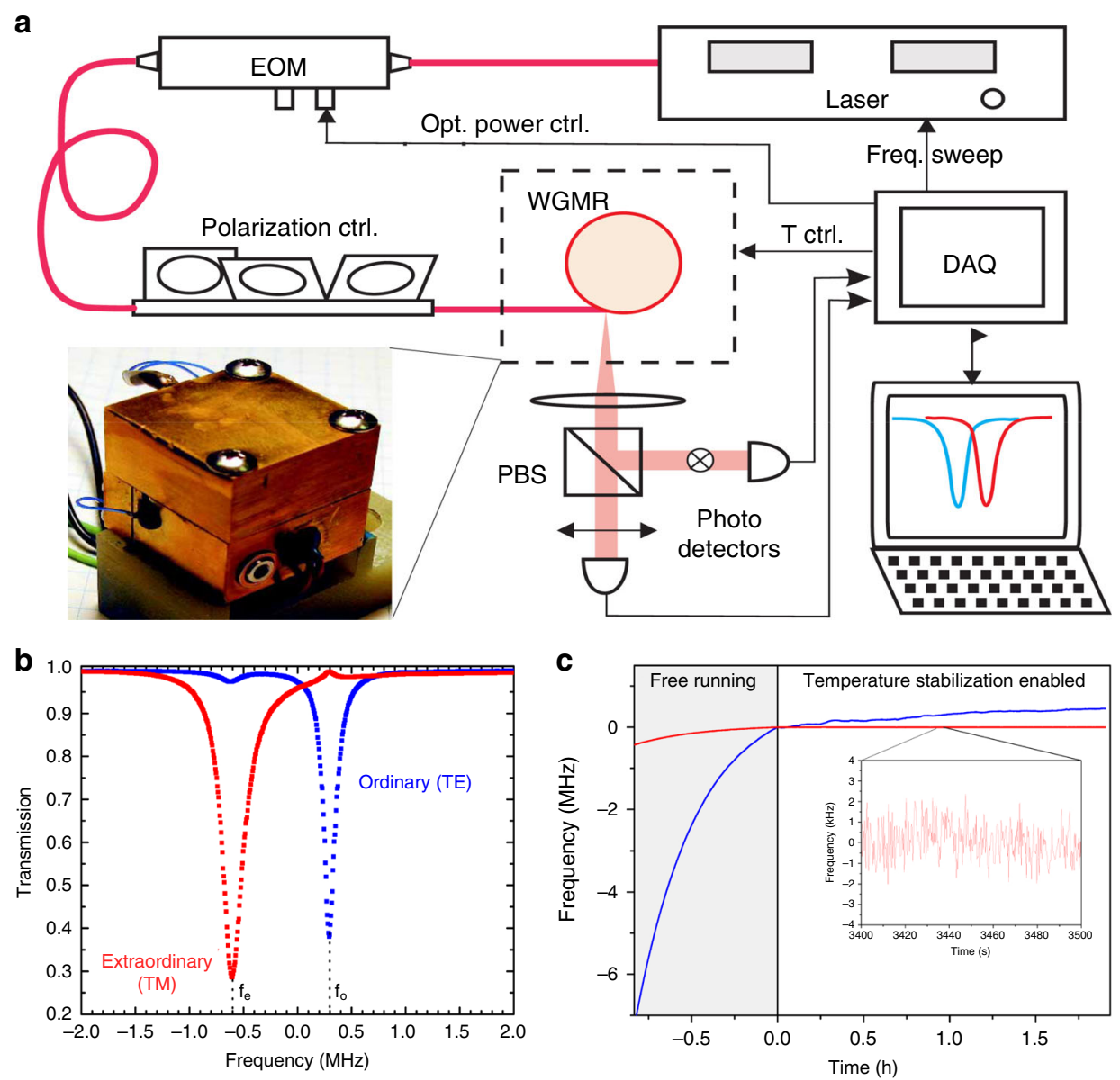

Fig. 11 Self-referenced dual-mode thermal stabilization technique. a The experimental setup of the self-referenced dual-mode stabilization technique ${ }^{136}$. b A pair of TE and TM WGMs with a small frequency detuning $\Delta f=f_{\mathrm{o}}-f_{\mathrm{e}}^{136}$. $\mathbf{c}$ The long-term absolute resonance frequency and dualmode frequency are shown as blue and red curves, respectively, in the free-running case $(t<0 \mathrm{~h})$ and the case with the temperature stabilized ( $t>$ 0 h) with the dual-mode technique. Inset: Magnification of the frequency fluctuation ${ }^{137}$. Reprinted with permission from ref. ${ }^{137}$ [OSA The Optical Society]

Experimentally, a millimeter-sized $\mathrm{MgF}_{2}$ disk resonator is excited by an angle-polished fiber coupler. The TE and TM modes are controlled by a fiber-based polarization controller, split by a polarizing beam splitter (PBS), and then monitored by two photodetectors, as shown in Fig. 11a. A data acquisition card is used to collect the signals of the photodetectors, which also provides feedback to an electro-optic modulator (EOM) to achieve the selfreferenced dual-mode stabilization process. A pair of TE and TM WGMs with a small frequency detuning are shown in Fig. $11 b^{136}$. The average frequency of these two resonances is utilized to locate the center of the next frequency sweep. The difference $\Delta f=f_{\mathrm{o}}-f_{\mathrm{e}}$ serves as the feedback error signal for temperature stabilization. Two feedback loops are used to achieve the temperature stabilization. The heaters in the brass cube are employed for the first feedback loop for long-term stabilization. An amplitude EOM with a high-speed heating effect is used to adjust the optical power, which provides short-term stabilization. Temperature stabilization on the order of a few nano-Kelvin was achieved (Fig. 11c).

\section{Conclusion}

In this review, we have briefly reviewed the mechanisms, techniques, and applications of thermo-optic dynamics in WGM microresonators. Thermal nonlinear effects, including both thermal bistability and thermal oscillation, have been discussed. Optothermal spectroscopy, thermal relaxation parameter measurement and optical thermal nonreciprocity in microresonators can be realized with the help of thermal bistability effects. Thermal tuning and thermal sensing are two examples of applications of the thermo-optic effect, which have been explored in various WGM resonator structures. By thermally scanning the resonance frequency of a microresonator, a singlefrequency laser with much lower noise and narrower linewidth can be used to reduce the noise of a frequency comb and an optical soliton. In addition, thermal locking 
techniques and photothermal imaging implemented with WGM microresonators have also been reviewed. Finally, we have introduced some techniques to achieve thermal stabilization, which is a critical prerequisite in many highperformance WGM applications.

The research field of thermo-optic dynamics in WGMs continues to grow. Looking ahead, more technological development and new applications will benefit from discoveries in this particular area. For example, the PDH locking technique could be exploited for thermal sensing by monitoring the feedback voltage signal ${ }^{140}$. A fast thermal scanning technique may find applications in optomechanics, nonlinear optics, optical trapping, sensing and imaging. Thermal stabilization techniques hold great potential for ultrastable microlasers by suppressing temperature fluctuations. Finally, resonator-assisted photothermal absorption spectroscopy and imaging techniques will open up new avenues to study materials and structure properties at the nanoscale. It is also worth noting that more opportunities will arise when materials with diverse thermo-optic properties are utilized in the resonators. Similar physics and mechanisms and the associated technologies could be explored with other optical resonant structures.

\section{Acknowledgements}

This work was supported by NSF grant nos. EFMA1641109 and ECCS1711451 and ARO grant no. W911NF1710189.

\section{Author contributions}

All authors contributed to the preparation of the manuscript.

\section{Conflict of interest}

The authors declare that they have no conflict of interest.

Received: 9 September 2019 Revised: 25 November 2019 Accepted: 20 December 2019

Published online: 24 February 2020

\section{References}

1. Vahala, K. J. Optical microcavities. Nature 424, 839-846 (2003).

2. Gaeta, A. L., Lipson, M. \& Kippenberg, T. J. Photonic-chip-based frequency combs. Nat. Photonics 13, 158-169 (2019).

3. Spencer, D. T. et al. An optical-frequency synthesizer using integrated photonics. Nature 557, 81-85 (2018).

4. Xiao, Y. F. et al. Tunneling-induced transparency in a chaotic microcavity. Laser Photonics Rev. 7, L51-L54 (2013).

5. Liu, Z. P. et al. High-Q asymmetric polymer microcavities directly fabricated by two-photon polymerization. Appl. Phys. Lett. 102, 221108 (2013).

6. Shao, L. B. et al. Ultrahigh-Q, largely deformed microcavities coupled by a free-space laser beam. Appl. Phys. Lett. 103, 121102 (2013).

7. Liu, Z. P. et al. Direct laser writing of whispering gallery microcavities by twophoton polymerization. Appl. Phys. Lett. 97, 211105 (2010).

8. Li, B. B. et al. Experimental observation of Fano resonance in a single whispering-gallery microresonator. Appl. Phys. Lett. 98, 021116 (2011).

9. Liu, Y. C. et al. Coupling of a single diamond nanocrystal to a whisperinggallery microcavity: photon transport benefitting from rayleigh scattering. Phys. Rev. A 84, 011805(R) (2011).

10. Li, B. B. et al. Experimental controlling of Fano resonance in indirectly coupled whispering-gallery microresonators. Appl. Phys. Lett. 100, 021108 (2012).
11. Liu, Y. C. et al. Cavity-QED treatment of scattering-induced free-space excitation and collection in high-Q whispering-gallery microcavities. Phys. Rev. A 85, 013843 (2012).

12. Jiang, X. F. et al. Chaos-assisted broadband momentum transformation in optical microresonators. Science 358, 344-347 (2017).

13. Lei, F. C. et al. Enhanced directional coupling of light with a whispering gallery microcavity. ACS Photonics (2020). https://doi.org/10.1021/ acsphotonics.9b01611.

14. Vollmer, F. \& Yang, L. Label-free detection with high-Q microcavities: a review of biosensing mechanisms for integrated devices. Nanophotonics 1, 267-291 (2012).

15. Wang, C. Q. et al. Electromagnetically induced transparency at a chiral exceptional point. Nat. Phys. (2020). https://doi.org/10.1038/s41567-019-07467.

16. Foreman, M. R., Swaim, J. D. \& Vollmer, F. Whispering gallery mode sensors. Adv. Opt. Photonics 7, 168-240 (2015).

17. Kippenberg, T. J. \& Vahala, K. J. Cavity optomechanics: back-action at the mesoscale. Science 321, 1172-1176 (2008).

18. Aspelmeyer, M., Kippenberg, T. J. \& Marquardt, F. Cavity optomechanics. Rev. Mod. Phys. 86, 1391-1452 (2014).

19. Peng, B. et al. Parity-time-symmetric whispering-gallery microcavities. Nat. Phys. 10, 394-398 (2014).

20. Wang, L. et al. Statistics of chaotic resonances in an optical microcavity. Phys. Rev. E 93, 040201(R) (2016).

21. Shu, F. J. et al. A scatterer-assisted whispering-gallery-mode microprobe. Nanophotonics 7, 1455-1460 (2018).

22. Yang, Q. F. et al. Dynamical tunneling-assisted coupling of high-Q deformed microcavities using a free-space beam. Phys. Rev. A 88, 023810 (2013).

23. Matsko, A. B. \& Ilchenko, V. S. Optical resonators with whispering-gallery modes - Part I: basics. IEEE J. Sel. Top. Quantum Electron. 12, 3-14 (2006).

24. Savchenkov, A. A. et al. Whispering-gallery-mode resonators as frequency references. II. Stabilization. J. Optical Soc. Am. B 24, 2988-2997 (2007).

25. Ilchenko, V. S. \& Gorodetskii, M. L. Thermal nonlinear effects in optical whispering gallery microresonators. Laser Phys. 2, 1004-1009 (1992).

26. Carmon, T., Yang, L. \& Vahala, K. J. Dynamical thermal behavior and thermal self-stability of microcavities. Opt. Express 12, 4742-4750 (2004).

27. Padmaraju, K. \& Bergman, K. Resolving the thermal challenges for silicon microring resonator devices. Nanophotonics 3, 269-281 (2014).

28. Chai, C. Z. et al. Thermal bistability of magnon in yttrium iron garnet microspheres. Appl. Phys. Lett. 114, 021101 (2019).

29. Lim, J. et al. Chasing the thermodynamical noise limit in whispering-gallerymode resonators for ultrastable laser frequency stabilization. Nat. Commun. $\mathbf{8}$, 8 (2017).

30. Grudinin, I. S. \& Vahala, K. J. Thermal instability of a compound resonator. Opt Express 17, 14088-14098 (2009).

31. Jiang, X. F. et al. Whispering gallery microsensors: a review. Preprint at https:// arxiv.org/abs/1805.00062 (2018).

32. Shao, L. B. et al. Detection of single nanoparticles and lentiviruses using microcavity resonance broadening. Adv. Mater. 25, 5616-5620 (2013).

33. Zhu, J. G. et al. On-chip single nanoparticle detection and sizing by mode splitting in an ultrahigh-Q microresonator. Nat. Photonics 4, 46-49 (2010).

34. Vollmer, F. et al. Protein detection by optical shift of a resonant microcavity. Appl. Phys. Lett. 80, 4057-4059 (2002).

35. Huang, S. H. et al. Whispering gallery mode resonator sensor for in situ measurements of hydrogel gelation. Opt. Express 26, 51-62 (2018).

36. $Y u, X . C$. et al. Single nanoparticle detection and sizing using a nanofiber pair in an aqueous environment. Adv. Mater. 26, 7462-7467 (2014).

37. Xu, X. Y. et al. Phone-sized whispering-gallery microresonator sensing system. Opt. Express 24, 25905-25910 (2016).

38. Baaske, M. D., Foreman, M. R. \& Vollmer, F. Single-molecule nucleic acid interactions monitored on a label-free microcavity biosensor platform. Nat. Nanotechnol. 9, 933-939 (2014).

39. Fan, X. D. \& Yun, S. H. The potential of optofluidic biolasers. Nat. Methods 11, 141-147 (2014).

40. Jiang, X. F. et al. Chip-based silica microspheres for cavity optomechanics. Opt. Express 23, 27260-27265 (2015).

41. Monifi, F. et al. Optomechanically induced stochastic resonance and chaos transfer between optical fields. Nat. Photonics 10, 399-405 (2016).

42. Dong, C. H. et al. Optomechanical dark mode. Science 338, 1609-1613 (2012).

43. Yang, L., Armani, D. K. \& Vahala, K. J. Fiber-coupled erbium microlasers on a chip. Appl. Phys. Lett. 83, 825-826 (2003). 
44. Jiang, X. F. et al. Whispering-gallery microcavities with unidirectional laser emission. Laser Photonics Rev. 10, 40-61 (2016).

45. He, L. N., Özdemir, Ş. K. \& Yang, L. Whispering gallery microcavity lasers. Laser Photonics Rev. 7, 60-82 (2013).

46. Jiang, X. F. et al. Highly unidirectional emission and ultralow-threshold lasing from on-chip ultrahigh-Q microcavities. Adv. Mater. 24, OP260-OP264 (2012).

47. Midya, B. et al. Supersymmetric microring laser arrays. Photonics Res. 7, 363-367 (2019).

48. Li, Y. H. et al. Whispering gallery mode microresonator for nonlinear optics. Preprint at: https://arxiv.org/abs/1809.04878 (2018).

49. Lin, G. P. et al. Nonlinear photonics with high-Q whispering-gallery-mode resonators. Adv. Opt. Photonics 9, 828-890 (2017).

50. Strekalov, D. V. et al. Nonlinear and quantum optics with whispering gallery resonators. J. Opt. 18, 123002 (2016).

51. Jiang, X. F. et al. Free-space coupled, ultralow-threshold Raman lasing from a silica microcavity. Appl. Phys. Lett. 103, 101102 (2013).

52. Yang, Y. et al. Four-wave mixing parametric oscillation and frequency comb generation at visible wavelengths in a silica microbubble resonator. Opt. Lett. 41, 5266-5269 (2016).

53. Huang, S. H. et al. Surface-enhanced Raman scattering on dielectric microspheres with whispering gallery mode resonance. Photon. Res. 6, 346-356 (2018).

54. Kasumie, S. et al. Raman laser switching induced by cascaded light scattering Laser Photonics Rev. 13, 1900138 (2019).

55. Peng, B. et al. Loss-induced suppression and revival of lasing. Science $\mathbf{3 4 6}$ 328-332 (2014).

56. Zhang, J. et al. A phonon laser operating at an exceptional point. Nat. Photonics 12, 479-484 (2018).

57. Zhu, J. G. et al. Optothermal spectroscopy of whispering gallery microresonators. Appl. Phys. Lett. 99, 171101 (2011).

58. Chen, W. J. et al. A simple method for characterizing and engineering thermal relaxation of an optical microcavity. Appl. Phys. Lett. 109, 061103 (2016).

59. Joshi, C. et al. Thermally controlled comb generation and soliton modelocking in microresonators. Opt. Lett. 41, 2565-2568 (2016).

60. Carmon, T. et al. Feedback control of ultra-high-Q microcavities: application to micro-Raman lasers and micro- parametric oscillators. Opt. Express $\mathbf{1 3}$ 3558-3566 (2005).

61. McRae, T. G. et al. Thermo-optic locking of a semiconductor laser to a microcavity resonance. Opt. Express 17, 21977-21985 (2009).

62. Heylman, K. D. et al. Optical microresonators as single-particle absorption spectrometers. Nat. Photonics 10, 788-795 (2016).

63. Knapper, K. A. et al. Single-particle photothermal imaging via inverted excitation through high-Q all-glass toroidal microresonators. Opt. Express $\mathbf{2 6}$ 25020-25030 (2018).

64. Knapper, K. A. et al. Chip-scale fabrication of high-Q all-glass toroidal microresonators for single-particle label-free imaging. Adv. Mater. 28, 2945-2950 (2016).

65. Liu, X. F. et al. Optothermal control of gains in erbium-doped whisperinggallery microresonators. Opt. Lett. 43, 326-329 (2018).

66. Wang, $\mathrm{T}$. et al. Optothermal control of the Raman gain enhanced ringing inmicroresonators. EPL (Europhys. Lett.) 124, 14002 (2018).

67. Wang, T. et al. Rapid and high precision measurement of opto-thermal relaxation with pump-probe method. Sci. Bull. 63, 287-292 (2018).

68. Sounas, D. L. \& Alù, A. Non-reciprocal photonics based on time modulation Nat. Photonics 11, 774-783 (2017).

69. Fan, L. et al. Silicon optical diode with $40 \mathrm{~dB}$ nonreciprocal transmission. Opt. Lett. 38, 1259-1261 (2013).

70. He, L. N. et al. Oscillatory thermal dynamics in high-Q PDMS-coated silica toroidal microresonators. Opt. Express 17, 9571-9581 (2009).

71. Luo, Z. C. et al. MHz-level self-sustained pulsation in polymer microspheres on a chip. AlP Adv. 4, 122902 (2014).

72. Deng, Y. et al. Thermo-optomechanical oscillator for sensing applications. Opt. Express 21, 4653-4664 (2013).

73. Fomin, A. E. et al. Nonstationary nonlinear effects in optical microspheres. J. Opt. Soc. Am. B 22, 459-465 (2005).

74. Park, Y. S. \& Wang, H. L. Regenerative pulsation in silica microspheres. Opt. Lett. 32, 3104-3106 (2007).

75. Deng, Y. et al. Thermo-optomechanical oscillations in high-Q ZBLAN microspheres. Opt. Lett. 38, 4413-4416 (2013).
76. Diallo, S., Lin, G. P. \& Chembo, Y. K. Giant thermo-optical relaxation oscillations in millimeter-size whispering gallery mode disk resonators. Opt. Lett. 40, 3834-3837 (2015).

77. Baker, C. et al. Optical instability and self-pulsing in silicon nitride whispering gallery resonators. Opt. Express 20, 29076-29089 (2012).

78. Wang, J. et al. Thermo-optic effects in on-chip lithium niobate microdisk resonators. Opt. Express 24, 21869-21879 (2016).

79. Sun, X. et al. Nonlinear optical oscillation dynamics in high-Q lithium niobate microresonators. Opt. Express 25, 13504-13516 (2017).

80. Yang, Y. et al. Tunable erbium-doped microbubble laser fabricated by sol-gel coating. Opt. Express 25, 1308-1313 (2017)

81. Linslal, C. L. et al. Tuning whispering gallery lasing modes from polymer fibers under tensile strain. Opt. Lett. 41, 551-554 (2016).

82. Chen, Y. et al. Tunable Raman laser in a hollow bottle-like microresonator. Opt. Express 25, 16879-16887 (2017).

83. Monifi, F. et al. A robust and tunable add-drop filter using whispering gallery mode microtoroid resonator. J. Lightwave Technol. 30, 3306-3315 (2012).

84. Aoki, T. et al. Observation of strong coupling between one atom and a monolithic microresonator. Nature 443, 671-674 (2006).

85. von Klitzing, W. et al. Frequency tuning of the whispering-gallery modes of silica microspheres for cavity quantum electrodynamics and spectroscopy. Opt. Lett. 26, 166-168 (2001).

86. Ward, J. M. \& Chormaic, S. N. Thermo-optical tuning of whispering gallery modes in Er: Yb co-doped phosphate glass microspheres. Appl. Phys. B: Lasers Opt. 100, 847-850 (2010).

87. Lee, B. S. et al. On-chip thermo-optic tuning of suspended microresonators. Opt. Express 25, 12109-12120 (2017).

88. Liu, S. et al. Strain-based tunable optical microresonator with an in-fiber rectangular air bubble. Opt. Lett. 43, 4077-4080 (2018).

89. Zhou, Z. H. et al. Broadband tuning of the optical and mechanical modes in hollow bottle-like microresonators. Opt. Express 25, 4046-4053 (2017).

90. Dinyari, K. N. et al. Mechanical tuning of whispering gallery modes over a $05 \mathrm{THz}$ tuning range with $\mathrm{MHz}$ resolution in a silica microsphere at cryogenic temperatures. Opt. Express 19, 17966-17972 (2011).

91. Yang, C. K. et al. Electrically tunable whispering gallery mode microresonator based on a grapefruit-microstructured optical fiber infiltrated with nematic liquid crystals. Opt. Lett. 42, 2988-2991 (2017).

92. Humar, M. et al. Electrically tunable liquid crystal optical microresonators. Nat. Photonics 3, 595-600 (2009).

93. Mur, M. et al. Magnetic-field tuning of whispering gallery mode lasing from ferromagnetic nematic liquid crystal microdroplets. Opt. Express $\mathbf{2 5}$ 1073-1083 (2017)

94. Shainline, J. M. et al. Broad tuning of whispering-gallery modes in silicon microdisks. Opt. Express 18, 14345-14352 (2010).

95. Henze, R. et al. Tuning whispering gallery modes using internal aerostatic pressure. Opt. Lett. 36, 4536-4538 (2011).

96. Henze, R., Ward, J. M. \& Benson, O. Temperature independent tuning of whispering gallery modes in a cryogenic environment. Opt. Express 21, 675-680 (2013).

97. Gil-Santos, E. et al. Scalable high-precision tuning of photonic resonators by resonant cavity-enhanced photoelectrochemical etching. Nat. Commun. 8 14267 (2017).

98. Liu, X. F. et al. Fabrication of a microtoroidal resonator with picometer precise resonant wavelength. Opt. Lett. 41, 3603-3606 (2016).

99. Koehler, L. et al. Direct thermo-optical tuning of silicon microresonators for the mid-infrared. Opt. Express 26, 34965-34976 (2018).

100. Kippenberg, T. J. et al. Dissipative Kerr solitons in optical microresonators. Science 361, eaan8083 (2018).

101. Yu, M. J. et al. Silicon-chip-based mid-infrared dual-comb spectroscopy. Nat. Commun. 9, 1869 (2018).

102. Dutt, A. et al. On-chip dual-comb source for spectroscopy. Sci. Adv. 4 e1701858 (2018)

103. Yu, M. J. et al. Mode-locked mid-infrared frequency combs in a silicon microresonator. Optica 3, 854-860 (2016).

104. Suh, M. G. et al. Microresonator soliton dual-comb spectroscopy. Science $\mathbf{3 5 4}$ 600-603 (2016).

105. Yang, Q. F. et al. Vernier spectrometer using counterpropagating soliton microcombs. Science 363, 965-968 (2019).

106. Okawachi, Y. et al. Octave-spanning frequency comb generation in a silicon nitride chip. Opt. Lett. 36, 3398-3400 (2011). 
107. Del'Haye, P. et al. Optical frequency comb generation from a monolithic microresonator. Nature 450, 1214-1217 (2007).

108. Yu, M. J. et al. Gas-phase microresonator-based comb spectroscopy without an external pump laser. ACS Photonics 5, 2780-2785 (2018).

109. Kessler, T. et al. A sub-40-mHz-linewidth laser based on a silicon single-crystal optical cavity. Nat. Photonics 6, 687-692 (2012).

110. Herr, T. et al. Temporal solitons in optical microresonators. Nat. Photonics $\mathbf{8}$, 145-152 (2014).

111. Yi, X. et al. Soliton frequency comb at microwave rates in a high-Q silica microresonator. Optica 2, 1078-1085 (2015).

112. Joshi, $\mathrm{C}$. et al. Counter-rotating cavity solitons in a silicon nitride microresonator. Opt. Lett. 43, 547-550 (2018).

113. Guan, G., Arnold, S. \& Otugen, M. V. Temperature measurements using a microoptical sensor based on whispering gallery modes. AIAA J. 44, 2385-2389 (2006).

114. Nam, S. H. \& Yin, S. Z. High-temperature sensing using whispering gallery mode resonance in bent optical fibers. IEEE Photonics Technol. Lett. 17, 2391-2393 (2005).

115. Guha, B., Cardenas, J. \& Lipson, M. Athermal silicon microring resonators with titanium oxide cladding. Opt. Express 21, 26557-26563 (2013).

116. Li, B. B. et al. On chip, high-sensitivity thermal sensor based on high-Q polydimethylsiloxane-coated microresonator. Appl. Phys. Lett. 96, 251109 (2010).

117. Dong, C. H. et al. Fabrication of high-Q polydimethylsiloxane optical microspheres for thermal sensing. Appl. Phys. Lett. 94, 231119 (2009).

118. Yan, Y. Z. et al. Packaged silica microsphere-taper coupling system for robust thermal sensing application. Opt. Express 19, 5753-5759 (2011).

119. Luo, R. et al. Self-referenced temperature sensing with a lithium niobate microdisk resonator. Opt. Lett. 42, 1281-1284 (2017).

120. Ioppolo, T. \& Rubino, E. Magnetic field-induced morphology-dependent resonances of a coupled composite metglas slab with a polymeric optical resonator. J. Polym. Sci. Part B: Polym. Phys. 52, 272-275 (2014).

121. $\mathrm{Xu}, \mathrm{L}$. H. et al. High-Q silk fibroin whispering gallery microresonator. Opt. Express 24, 20825-20830 (2016).

122. Wang, Y. et al. Tapered optical fiber waveguide coupling to whispering gallery modes of liquid crystal microdroplet for thermal sensing application. Opt. Express 25, 918-926 (2017).

123. Liu, Z. H. et al. Whispering gallery mode temperature sensor of liquid microresonastor. Opt. Lett. 41, 4649-4652 (2016).

124. Ward, J. M., Yang, Y. \& Chormaic, S. N. Highly sensitive temperature measurements with liquid-core. IEEE Photonics Technol. Lett. 25, 2350-2353 (2013).
125. Chen, X. G. et al. Packaged droplet microresonator for thermal sensing with high sensitivity. Sensors 18, 3881 (2018).

126. Ilchenko, V. S. et al. Nonlinear optics and crystalline whispering gallery mode cavities. Phys. Rev. Lett. 92, 043903 (2004).

127. Wu, R. B. et al. Lithium niobate micro-disk resonators of quality factors above 107. Opt. Lett. 43, 4116-4119 (2018).

128. Black, E. D. An introduction to Pound-Drever-Hall laser frequency stabilization. Am. J. Phys. 69, 79-87 (2001).

129. Suter, J. D. et al. Thermal characterization of liquid core optical ring resonator sensors. Appl. Opt. 46, 389-396 (2007).

130. Han, M. \& Wang, A. B. Temperature compensation of optical microresonators using a surface layer with negative thermo-optic coefficient. Opt. Lett. 32, 1800-1802 (2007).

131. He, L. et al. Compensation of thermal refraction effect in high- $Q$ toroidal microresonator by polydimethylsiloxane coating. Appl. Phys. Lett. 93, 201102 (2008)

132. Xie, C. F. et al. Compensation of thermal nonlinear effect in hybrid microsphere resonators. Micro Nano Lett. 9, 609-612 (2014).

133. Zhi, Y. \& Meldrum, A. Tuning a microsphere whispering-gallery-mode sensor for extreme thermal stability. Appl. Phys. Lett. 105, 031902 (2014).

134. Kim, E. et al. Thermal characterisation of (bio)polymers with a temperaturestabilised whispering gallery mode microsensor. Appl. Phys. Lett. 106, 161101 (2015).

135. Grudinin, I. et al. Compensation of thermal nonlinearity effect in optical resonators. Opt. Express 19, 7365-7372 (2011).

136. Strekalov, D. V. et al. Temperature measurement and stabilization in a birefringent whispering gallery mode resonator. Opt. Express 19, 14495-14501 (2011)

137. Fescenko, I. et al. Dual-mode temperature compensation technique for laser stabilization to a crystalline whispering gallery mode resonator. Opt. Express 20, 19185-19193 (2012).

138. Baumgartel, L. M., Thompson, R. J. \& Yu, N. Frequency stability of a dual-mode whispering gallery mode optical reference cavity. Opt. Express 20, 29798-29806 (2012).

139. Weng, W. L., Anstie, J. D. \& Luiten, A. N. Refractometry with ultralow detection limit using anisotropic whispering-gallery-mode resonators. Phys. Rev. Appl. 3, 044015 (2015).

140. Swaim, J. D., Knittel, J. \& Bowen, W. P. Detection of nanoparticles with a frequency locked whispering gallery mode microresonator. Appl. Phys. Lett. 102, 183106 (2013). 